\title{
Selection of Acid Tolerant Purple Nonsulfur Bacteria for Application in Agriculture
}

Phitthaya Nookongbut ${ }^{1}{ }^{*}$, Nawaporn Jingjit ${ }^{1}$, Duangporn Kantachote ${ }^{1}$, Ampaitip Sukhoom ${ }^{1}$, and Manee Tantirungkij ${ }^{2}$

${ }^{1}$ Department of Microbiology, Faculty of Science, Prince of Songkla University, Songkhla 90112, Thailand

${ }^{2}$ Research and Academic Service Center, Faculty of Agriculture and Kamphaeng Saen, Kasetsart University, Kamphaeng Saen Campus, Nakhon Pathom 73140, Thailand

*Corresponding author. E-mail: phitthaya.nookongbut@gmail.com https//:doi.org/10.12982/CMUJNS.2020.0049

Received: August 8, 2019 Revised: November 22, 2019 Accepted: December 18, 2019

\section{ABSTRACT}

To screen acid tolerant purple nonsulfur bacteria (PNSB) isolated from peat swamp forests for their abilities to produce plant growth promoting substances (aminolevulinic acid: ALA, siderophores, indole-3-acetic acid: $I A A)$ and also release ammonium from $N_{2}$ fixation. Among 10 PNSB isolates grown in basic isolation medium (BIM), pH 4.5 found that strain KKSSR91 was the most effective to release ALA and siderophores under microaerobic light conditions; while strain KTPWG11 exhibited highest production of siderophores under aerobic dark conditions. Based on ALA production in medium containing $60 \mathrm{mM}$ glycine, 4 PNSB isolates were selected for further studies and found that strain KTSSR92 produced highest IAA at $36.9 \mathrm{mg} / \mathrm{L}$. All of them were able to release $\mathrm{NH}_{4}^{+}$, but no activity to solubilize phosphate. In addition, maximal ALA production in the medium supplemented with glycine by strain KKSSR91 was $2.89 \mathrm{mg} / \mathrm{L}$ at 4000 lux light intensity. Seed germination index (GI) of kidney bean (Phasecolus vulgaris L.) test using strain KKSSR91 at $1 \mathrm{~g}$ fresh biomass $/ \mathrm{L}, \mathrm{pH} 4.5$ was $68.81 \%$ compared with a control in distilled water as only 58.68\%. Strain KKSSR91 was identified using 16S rRNA sequencing and found that it showed $99 \%$ similarity with Rhodopseudomonas palustris.

Keywords: 5-aminolevulinic acid, Germination index, Indole-3-acetic acid, Plant growth promoter, Rhodopseudomonas palustris, Siderophores 


\section{INTRODUCTION}

More than $50 \%$ of the world's potential arable land is estimated to be acidic soils based on pH lower than 5.5 (Bot et al., 2000). Bioavailability is limited in acid soils and the subsequent stunted development of crops contributes to the need for fertilizers (Yang et al., 2013). Among critical factors, phytotoxicity due to heavy metals (mostly $\mathrm{Fe}, \mathrm{Al}$ and $\mathrm{Mn}$ ) is the major obstruction to agricultural production on acid sulfate soils (Khuong et al., 2017; Nguyen et al., 2018). Several effective techniques have been applied to overcome this serious problem. Lime and fertilizer amendments, particularly phosphate, are routinely applied to acidic soils for agriculture. Recently, plant growth promoting bacteria (PGPB) have gained a lot of attention worldwide for their ability to enhance agricultural production. To cope with stress caused by low $\mathrm{pH}$, purple nonsulfur bacteria (PNSB), especially Rhodopseudomonas palustris, release several plant growth promoting substances (PGPS) using a variety of mechanisms (Sakpirom et al., 2017; Khuong et al., 2018). This behavior of PNSB encouraged us to visit peatlands in Thailand to isolate and obtain several strains of $R$. palustris that exhibited the plant growth promoting characters of iron sequestration (siderophores), metal entrapment (exopolymeric substances), nitrogen fixation, phosphate solubilization, 5-aminolevulinic acid (ALA) synthesis, and indole-3acetic acid (IAA) formation (Nookongbut et al., 2019).

$R$. palustris strains not only promote growth in plants but also induce resistance to stresses from sodium and heavy metals (Nunkaew et al., 2014; Kantachote et al., 2016; Sakpirom et al., 2017; Nookongbut et al., 2018). For instance, $R$. palustris released ALA to alleviate the effects of $\mathrm{NaCl}$ on rice growth (Nunkaew et al., 2014; 2015a, b). In addition to ALA, other PGPS could promote rice growth under arsenic (As) stress by increasing chlorophylls to support both antioxidant scavenging enzymes and non-enzymatic systems that exclude As species from rice (Nookongbut et al., 2018). The kidney bean (Phaseolus vulgaris L.) is a major food legume that provides proteins and essential amino acids to several million people (Rangel et al., 2005). Normally, the kidney bean grows best in a pH range from 6.0 to 6.8. However, acidic soils inhibit bean growth and severe weak to acid soils ( $\mathrm{pH} 4.5$ to 5.5) (Alemu, 2017). Therefore, the kidney bean could be considered a plant indicator of acid stress. The present study aimed to investigate the plant growth promoting characteristics of acid tolerant $R$. palustris strains and their ability to alleviate the effects of acidic stress on kidney bean seed germination. Hence, our study could aid the further development and application of $R$. palustris as a PGPB for sustainable agriculture on acidic arable land. 


\section{MATERIALS AND METHODS}

\section{Inoculum preparation}

This study used ten acid tolerant PNSB strains, KTPSG1, KTPSG2, KTSWR2, KTPWG11, KTPWR12, KTSWR13, KKSWG14, KTSSR19, KTSSR92, and KKSSR91, previously isolated from peat swamp forests in Southern Thailand (Nookongbut et al., 2019). To prepare the active culture, each PNSB strain was sub-cultured twice and one loopful of each was inoculated into $12 \mathrm{ml}$ acidic BIM (Basic Isolation Medium) broth at $\mathrm{pH}$ 4.5. Active cultures were incubated for $48 \mathrm{~h}$ under microaerobic light condition with a little space in the test tube under light intensity of 3,000 lux. To obtain a cell pellet, the culture broth was centrifuged at $8,000 \mathrm{rpm}$ for $15 \mathrm{~min}$. The cell suspension was prepared with normal saline solution (NSS) and adjusted turbidometrically at a wavelength of $660 \mathrm{~nm}$ at 0.6 to obtain an inoculum with a cell count of approximately $\sim 10^{8}$ $\mathrm{CFU} / \mathrm{ml}$. A ten percent inoculum size was used for all experiments in this study, unless otherwise stated.

\section{Characterization of PGP traits}

The growth-promoting features of acid tolerant PNSB were characterized by their production of ALA, IAA, siderophores, nitrogen-fixing based on ammonium ion release and phosphate-solubilization. PNSB grow as phototrophs or heterotrophs depending on the environmental conditions of light and oxygen (Madigan and Jung 2009). Therefore, almost all our experiments were carried out in parallel in microaerobic light and aerobic dark conditions. The microaerobic light condition was the same condition used for the inoculum preparation. Otherwise, different light intensities are stated. The aerobic dark condition was set by growing PNSB in a medium volume equal to $40 \%$ of the test tube volume in an incubator with shaking at certain speeds.

The siderophores-producing PNSB were tested with a slightly modified Chrome Azurol Sulfonate (CAS) Assay as described by Nookongbut et al. (2018). Three wells were created in a triangle on a CAS agar plate, and each well was inoculated with a $300 \mu \mathrm{l}$ PNSB inoculum. All agar plates were incubated under microaerobic light and aerobic dark conditions for 5 days. Siderophores production was evaluated by measuring the diameter of clear zones around the wells.

ALA content was determined according to the modified Ehrlich's method of Burnham (1970). PNSB were grown in BIM broth supplemented with $60 \mathrm{mM}$ glycine (Merck KGaA, GR for analysis) (Sasaki et al., 1990), under microaerobic light conditions only for 5 days. Glycine and succinyl-CoA are precursors for ALA synthesis by PNSB that use C4 (Shemin pathway) (Avissar and Moberg, 1995). However, this study focused only on glycine, due to the fact that succinyl-CoA is an intermediate in the Krebs cycle and in the conversion of propionly-CoA to methylmalonyl-CoA and then to succinyl-CoA. This approach 
is also supported by Sasaki et al., (1990) who reported that adding succinate did not increase ALA production by PNSB. Harvested cells by centrifugation at 8,000 rpm, for $20 \mathrm{~min}, 2 \mathrm{ml}$ of supernatant were mixed with $1 \mathrm{ml}$ of $1 \mathrm{M}$ sodium acetate (Merck KGaA, GR for analysis) buffer ( $\mathrm{pH} \mathrm{4.7)}$ and $0.5 \mathrm{ml}$ of acetylacetone (2,4-pentanedione) (Sigma-Aldrich,AR) in screw cap tubes. The mixture was then boiled at $100{ }^{\circ} \mathrm{C}$ in a water bath for $15 \mathrm{~min}$. After allowing the reaction mixture to cool to room temperature, $3.5 \mathrm{ml}$ of Ehrlich's reagent was added to the tube and left for $30 \mathrm{~min}$. Using a spectrophotometer at a wavelength of $554 \mathrm{~nm}$, the reaction mixture was determined colorimetrically and the content of ALA was calculated using an ALA standard curve generated with dilutions of commercial ALA (Sigma-Aldrich Inc., PREMIUM s $\geq 98 \%$ ).

Ammonium release was examined in terms of $\mathrm{N}_{2}$ fixation by cultivation in a nitrogen-free medium of BIM broth at $\mathrm{pH}$ 4.5. This experiment used four selected PNSB strains (KKSSR91, KTPWG11, KTSSR92 and KTSSR19) which performed best in the siderophores-production and ALA tests. The four strains were cultured in the designed medium and incubated under microaerobic light and aerobic dark conditions for four days. A qualitative $\mathrm{NH}_{4}{ }^{+}$assay was carried out using $0.5 \mathrm{ml}$ of cell-free culture medium from each strain mixed with 2 to 3 drops of Nessler's reagent (Sigma-Aldrich, AR). The presence of $\mathrm{NH}_{4}{ }^{+}$was evaluated by observation of color changes: no color change indicated an absence of $\mathrm{NH}_{4}{ }^{+}$; while light yellow, yellow, and brown indicated low, medium and high levels of $\mathrm{NH}_{4}^{+}$, respectively. Uninoculated medium served as a control and was tested in the same conditions as the medium inoculated with PNSB.

IAA production was measured using a slightly modified version of Salkowski's reagent test (Gordon and Weber, 1951). The four previously selected PNSB strains were cultivated in BIM broth medium supplemented with $3 \mathrm{mM} \mathrm{L-}$ tryptophan (Merck, GR), at $\mathrm{pH} 4.5$ under microaerobic light conditions only for 5 days. To obtain a supernatant for IAA content determination, PNSB cells were harvested from the BIM culture broth by centrifugation at 8,000 rpm for $15 \mathrm{~min}$. One milliliter of the culture supernatant was placed in a test tube and mixed with $2 \mathrm{ml}$ Salkowski's reagent. The mixture was incubated at room temperature for $30 \mathrm{~min}$. The reddish color resulting from the mixture reaction was measured using a spectrophotometer at a wavelength of $535 \mathrm{~nm}$. The level of IAA was calculated based on the standard curve of authentic IAA (Sigma-Aldrich, AR) concentrations.

To measure phosphate solubilization, $300 \mu 1$ of each PNSB inoculums was introduced by micropipette into wells $(0.8 \mathrm{~mm})$ in modified BIM agar medium supplemented with $4.5 \mathrm{~g} / \mathrm{L}$ each of the insoluble phosphates $\mathrm{AlPO}_{4}$ and $\mathrm{FePO}_{4}$ (Sigma-Aldrich, AR) (Nookongbut et al., 2019). The $\mathrm{pH}$ was adjusted to 4.5. After incubation for 7 days under microaerobic light and aerobic dark conditions, the ability of each strain to solubilize phosphate was determined based on the presence of clear zones surrounding the wells in agar plates. 


\section{Optimization conditions for ALA production}

Since strain KKSSR91 performed best in the experiment for ALA production, the conditions of ALA production were optimized using only this strain. KKSSR91 was cultivated in BIM broth at $\mathrm{pH} 4.5$ supplemented with $60 \mathrm{mM}$ glycine. In the microaerobic light condition, the effect of light intensity was investigated at 3,000, 4,000 and 5,000 lux. In the aerobic dark condition, the effect of shaking was evaluated by growing strain KKSSR91 in incubator shakers at speeds of $0,50,100$ and $150 \mathrm{rpm}$. All treatments were incubated at room temperature for 5 days. ALA content was analyzed using the modified Ehrlich's method as previously described by Burnham (1970).

\section{Effect of PNSB on seed germination under acidic stress}

The kidney bean (Phasecolus vulgaris L.) was used to investigate seed germination under acidic stress. All seeds were washed three times with tap water, followed by $70 \%$ ethanol (RCI Labscan, AR) for $30 \mathrm{~s}$ and then surface-sterilized in $1 \%$ sodium hypochlorite solution (Loba Chemie Laboratory, Reagents) for $10 \mathrm{~min}$. The beans were rinsed 5 times with distilled water (DW). Seed germination was tested on a wet filter paper, with $10 \mathrm{ml}$ of controls/treatments. Strain KKSSR91 was grown in BIM broth at $\mathrm{pH} 4.5$ under light for 2 days. The broth was centrifuged to obtain a cell pellet to prepare the required cell suspension. The experiment was designed to test the effect of PNSB on seed germination under acidic stress. Eight sets $(2 \times 4)$ were designed as follows: two controls of uninoculated PNSB (DW, $\mathrm{pH} 7$ and DW, $\mathrm{pH} 4.5$ ); six sets of inoculated PNSB in biomass doses of 1,5 and $10 \mathrm{~g}$ wet biomass/L under neutral and acidic stress conditions. The aim was to use PNSB as a PGPB in a seed soaking step since the PNSB were to remain active until planting, so wet biomass was chosen for the test. Ten kidney bean seeds were placed in each plate, covered with a lid, and incubated in darkness at room temperature $\left(25 \pm 3{ }^{\circ} \mathrm{C}\right)$. After 5 days, the numbers of germinated seed were counted and their shoot heights and root lengths were measured. The results were expressed as percent germination by comparing a DW, pH 7.0 set. The percentage of relative seed germination (RSG), relative root growth (RRG), and the germination index (GI) were determined following the method described by Kantha et al., (2015). Each set consisted of three replicates $(3 \times 10, \mathrm{n}=30)$ and values were calculated from the following equations:

$$
\begin{gathered}
\text { RSG }(\%)=\frac{\text { Number of seeds germinated in the KKSSR } 91}{\text { Number of seeds germinated in distilled water }} \times 100 \\
\operatorname{RRG~}(\%)=\frac{\text { Mean root length in the presence of } K K S S R 91}{\text { Mean root length in distilled water }} \times 100 \\
\mathrm{GI}(\%)=\frac{\text { RSG } \mathrm{RRG}}{100}
\end{gathered}
$$




\section{Bacterial identification and phylogenetic analysis}

16S rRNA genes were analyzed to identify the selected PNSB strain KKSSR91 in the following procedure. DNA was extracted using a DNA extraction kit (TIANGEN ${ }^{\circledR}$ DNA extraction kit, China) according to the manufacturer's instructions. Nearly full length $16 \mathrm{~S}$ rRNA genes $(\sim 1,500 \mathrm{bp})$ were amplified using universal primers: $1,492 \mathrm{r}$ primer (5'-TACCTTGTTACGACTT) and $27 \mathrm{f}$ primer (5'-AGAGTTTGATCCTGGCTCAG-3'). The thermal cycling (Bio-Rad) protocol used the following parameters: initial denaturation at $94{ }^{\circ} \mathrm{C}$ for $1 \mathrm{~min}$, followed by 35 cycles of denaturation at $95{ }^{\circ} \mathrm{C}$ for $1 \mathrm{~min}$, annealing at $55^{\circ} \mathrm{C}$ for $1 \mathrm{~min}$, and extension, at $72{ }^{\circ} \mathrm{C}$ for $1 \mathrm{~min}$, plus a final extension step at $72{ }^{\circ} \mathrm{C}$ for $10 \mathrm{~min}$. The amplified PCR products were purified with a PCR Purification Kit (BioFACT ${ }^{\mathrm{TM}}$. PCR Purification Kit, Korea), and sequences were analyzed at the Macrogen sequencing facility (Macrogen, Seoul, Korea). To establish the similarity of $16 \mathrm{~S}$ rRNA genes, sequence data were analyzed using the public database, NCBI nucleotide BLAST. Identification of the PNSB isolate was followed by the construction of a phylogenetic tree. The 16S rRNA sequences were aligned using the ClustalW alignment software, while the distance analysis and construction of the phylogenetic tree were performed using the MEGA version $X$ program. Substitution patterns and rates were estimated using the Tamura and Nei model (+G). Maximum Likelihood (ML) estimation of the gamma parameter was used to evaluate the sequence distance among strains, and the phylogeny was analyzed using the Bootstrap method with 1,000 repetitions to estimate tree reliability. Pseudomonas aeruginosa type strain DSM $50071^{\mathrm{T}}$ were used as an out-group in this phylogenetic tree construction.

\section{Statistical analysis}

One-way analysis of variance (ANOVA) was used for data analysis. The results are shown as their means \pm standard deviations, and significant differences were evaluated using the Duncan's Multiple Range Test at the level $P<0.05$. Statistical analysis was performed using the statistical package for the Social Sciences 15.0 software for Windows (SPSS).

\section{RESULTS}

\section{Characterization of acid tolerant PNSB for beneficial traits}

The acid stress tolerance potential of 10 PNSB isolates was evaluated from their PGP characteristics. Production levels of ALA were compared between bacteria grown in acidic BIM broth without the addition of glycine as a control and in the same medium with a precursor of glycine at $60 \mathrm{mM}$ (Figure 1). Under microaerobic light condition, the released ALA concentration, determined by colorimetry, ranged from 0.25 to $2.41 \mathrm{mg} / \mathrm{L}$. Only four PNSB strains produced good levels of ALA. Ranked by ALA production these were in the order KKSSR91 > KTPWG11 > KKSWG19 KTSSR92. The highest concentration 
of released ALA was $2.41 \mathrm{mg}$ ALA/L. This level was produced by strain KKSSR91 in BIM broth supplemented with $60 \mathrm{mM}$ glycine. The high ALA concentrations produced by most strains in the presence of glycine (Figure 1) shows the benefit of using the precursor.

All the PNSB isolates tested released siderophores in both incubating conditions. In the microaerobic light condition, siderophores were released in a range from 1.06 to $1.41 \mathrm{~cm}$ and in the aerobic dark condition from 0.9 to $1.40 \mathrm{~cm}$ (Figure 2). The strains KKSSR91 and KTPWG11 released the highest levels of siderophores (Figure 2 and 3). In the case of KKSSR91, no significant difference in released siderophores was observed between incubating conditions but strain KTPWG11 released a significantly higher level of siderophores in the aerobic dark condition than in the microaerobic light condition (Figure 2). However, except for strain KKSSR91, all tested PNSB released more siderophores in the microaerobic light condition.

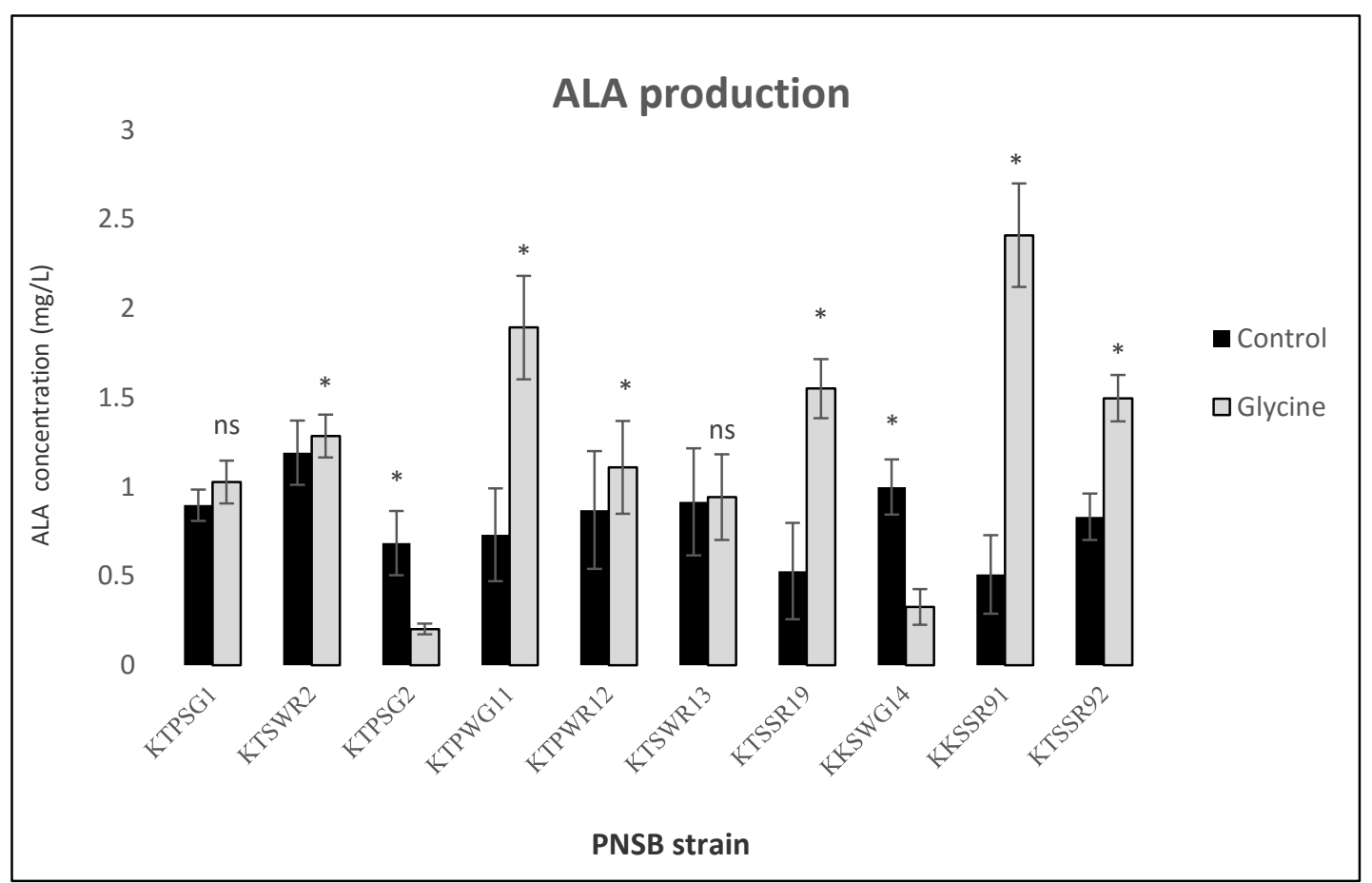

Figure 1. Effect of glycine on ALA production by acid tolerant PNSB cultured in BIM medium containing $0.60 \mathrm{mM}$ glycine, $\mathrm{pH} 4.5$ under microaerobic light conditions for 5 days. Asterisk denotes significant differences $(P<0.05)$ compared with control. 


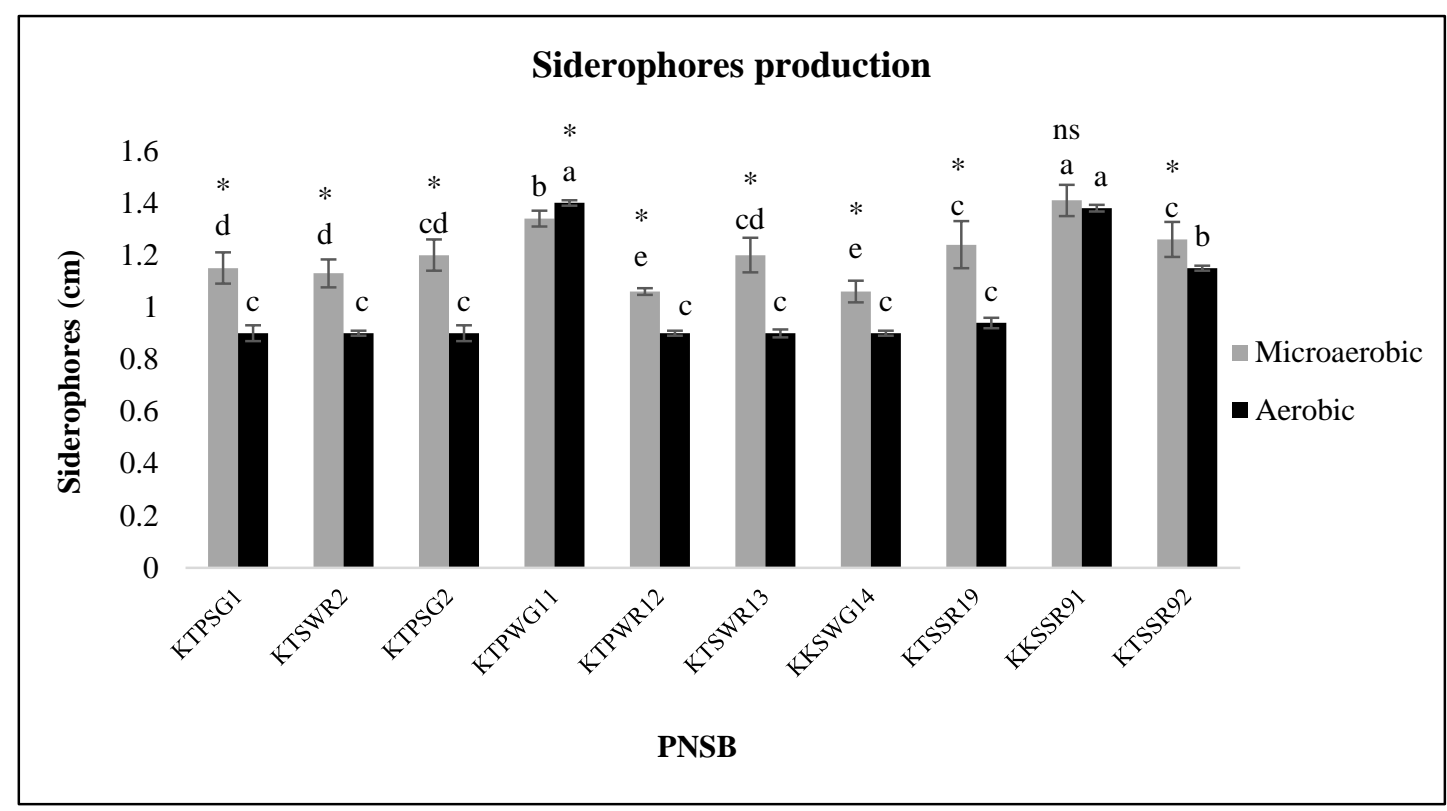

Figure 2. Siderophores production by acid tolerant PNSB based on clear zone around wells in BIM medium, $\mathrm{pH} 4.5$ under microaerobic light and aerobic dark conditions for 5 days. Different lowercase alphabets denote significant differences $(P<0.05)$, and asterisk denotes significant differences $(P<0.05)$ between both incubating conditions; ns: no significant difference.

Based on the results of the first two experiments, four PNSB strains were selected for further studies. The $\mathrm{N}_{2}$-fixing ability of the selected strains was determined based on released ammonium ion in a nitrogen-free medium under acidic stress. With the exception of strain KTPWG11 grown in the microaerobic light conditions, culture supernatants of all strains grown under both incubating conditions developed a light yellow color after the addition of Nessler's reagent (Table 1). The release of ammonium ion by KTPWG11 in the microaerobic light condition only indicated $\mathrm{N}_{2}$-fixation at the medium level. All four strains were therefore classified as low $\mathrm{N}_{2}$-fixers.

Table 1. Nitrogen fixation on the basis of released ammonium ion by selected acid tolerant PNSB in nitrogen free medium under acid stress at $\mathrm{pH} 4.5$.

\begin{tabular}{ccc}
\hline PNSB & Microaerobic light & Aerobic dark \\
\hline KTPWG11 & ++ & + \\
KTSSR19 & + & + \\
KKSSR91 & + & + \\
KTSSR92 & + & + \\
\hline Note: +: responded on Nessler's reagent as light yellow (low); ++: yellow (medium); +++: brown (high).
\end{tabular}




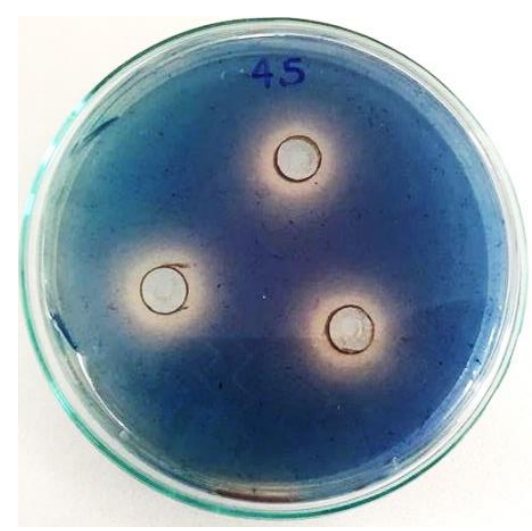

A1

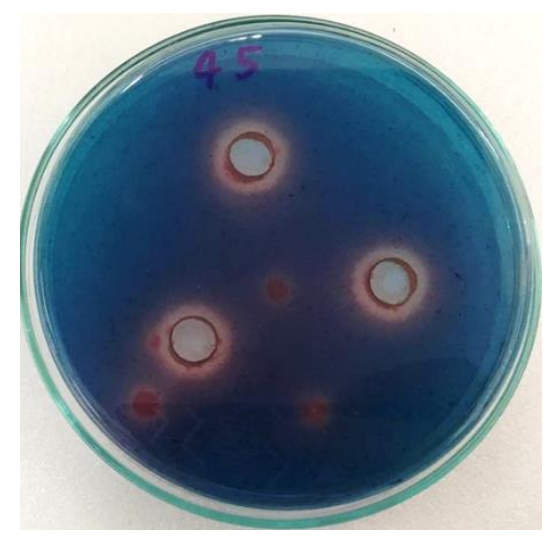

A2

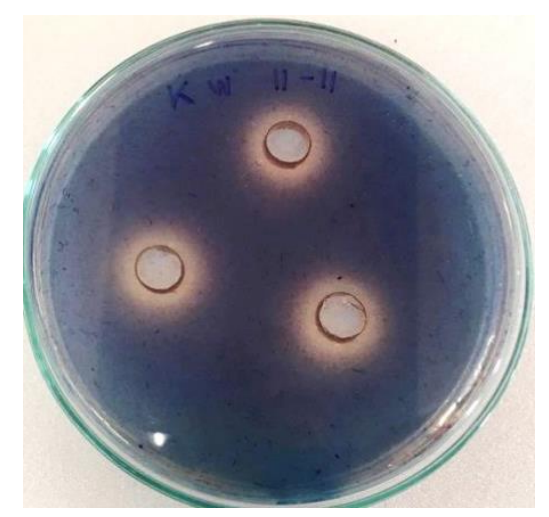

B1

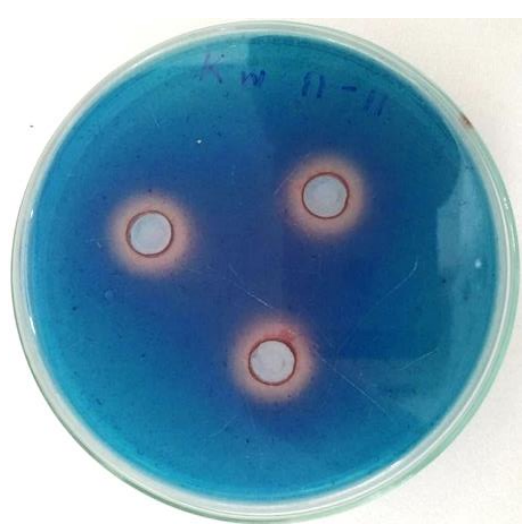

B2

Figure 3. Pictures of siderophores produced by acid tolerant PNSB based on clear zone around wells in BIM medium, $\mathrm{pH} 4.5$ under incubating conditions; 1. Microaerobic light 2. Aerobic dark for 5 days (A: KKSSR91 and B: KTPWG11).

In the microaerobic light condition in acidic BIM broth supplemented with $3 \mathrm{mM}$ L-tryptophan, the four selected PNSB secreted IAA in a range of 26.46 to $36.9 \mathrm{mg} / \mathrm{L}$ (Figure 4). The release of IAA was ranked in the order KTSSR92 > KKSWG19 > KKSSR91 > KTPWG11. However, under acidic stress, all four strains were unable to solubilize phosphate in both incubating conditions. 


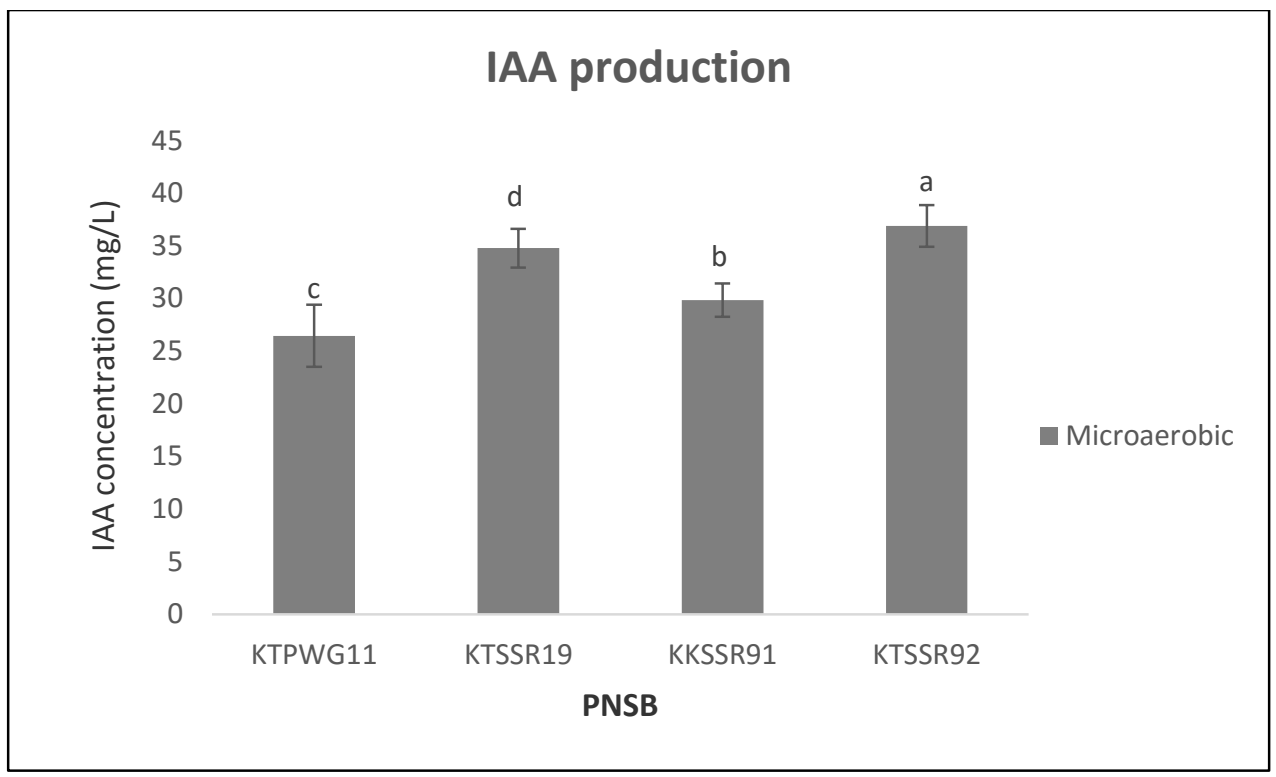

Figure 4. IAA production by acid tolerant PNSB strain KKSSR91 in BIM broth containing $3 \mathrm{mM}$ L-tryptophan, $\mathrm{pH} 4.5$ under microaerobic light conditions for 5 days. Different lowercase alphabets denote significant differences $(P<0.05)$.

\section{Optimization conditions for ALA production}

According to the results of the previous experiments, strain KKSSR91 most effectively released both ALA and siderophores under both incubating conditions (Figure 1 and 2). Therefore, the effect of different growth conditions on ALA production was investigated using only strain KKSSR91. When the effect of agitation speed in the aerobic dark condition was investigated, no significant difference was found between speeds. In fact, the static condition produced more ALA than shaking (50,100 and $150 \mathrm{rpm}$ ) (Table 2). Under aerobic dark condition, the highest concentration of ALA released was $1.28 \mathrm{mg} / \mathrm{L}$, which occurred in the static condition. In the microaerobic light condition, there was no significant difference in ALA production $(2.46-2.89 \mathrm{mg} / \mathrm{L})$ at different light intensities $(3,000,4,000$ and 5,000) (Table 2). The highest level of ALA produced by KKSSR91 ( $2.89 \mathrm{mg} / \mathrm{L})$ occurred at 4,000 lux. 
Table 2. Effects of agitation speed and light intensity on ALA production under acidic stress by strain KKSSR91 in GM containing $60 \mathrm{mM}$ glycine for 5 days.

\begin{tabular}{cc}
\hline $\begin{array}{c}\text { Agitation speed } \\
(\mathbf{r p m})\end{array}$ & $\begin{array}{c}\text { Aerobic dark } \\
\text { ALA }(\mathbf{m g} / \mathbf{L})\end{array}$ \\
\cline { 2 - 2 } 0 & $1.28 \pm 0.15$ \\
50 & $0.66 \pm 0.74$ \\
100 & $0.99 \pm 0.46$ \\
150 & $0.94 \pm 0.48$ \\
\hline Significance & ns \\
\hline Light intensity & Microaerobic light \\
(lux) & ALA (mg/L) \\
3000 & $2.53 \pm 0.29$ \\
4000 & $2.89 \pm 0.77$ \\
5000 & $2.46 \pm 0.98$ \\
\hline Significance & $\mathrm{ns}$ \\
\hline
\end{tabular}

Note: Value is mean of three determinations \pm standard deviation (S.D.); ns: no significant difference at $P>0.05$.

\section{Effect of PNSB on Seed germination of kidney bean under acidic stress}

Due to its effective production of ALA, siderophores and IAA, KKSSR91 was selected to investigate the potential amelioration of acidic stress on kidney bean seed germination. The performance of the strain was determined on the basis of GI index (\%) and the results are shown in Table 3 . The control set at $\mathrm{pH} 7$ showed a maximum GI index of $100 \%$, but under acidic stress at $\mathrm{pH} 4.5$ the GI index of the control was only $58.68 \%$. In contrast, in the neutral $\mathrm{pH}$ condition, the GI index of all inoculated sets was lower than the control at $\mathrm{pH}$ 7.0. However, under acidic stress the GI index of every PNSB-inoculated set $(61.92-68.81 \%)$ was higher than the control (58.68\%). At higher biomass doses seed germination reduced, so for a biomass dose of $1 \mathrm{~g} / \mathrm{L}$ was considered suitable for both neutral and acidic conditions as the obtained GI indices were 84.87 and $68.81 \%$, respectively.

\section{Bacterial identification and phylogenetic analysis}

The acid-resistant PNSB strain KKSSR91 was isolated from soil collected from a secondary peat swamp forest at Kuan Kreng in Nakorn Si Thammarat province, Thailand (Nookongbut et al., 2019). It was identified for its phylogenetic assignment based on partial molecular 16S rRNA gene ( 1,500 bp) sequencing profiles. The Maximum Likelihood-based phylogenetic tree is 
presented in Figure 5. The sequences were aligned with sequences retrieved from validated $R$. palustris species. Phylogenetic analysis was conducted using the Maximum Likelihood method with bootstrapping for 1,000 replicates. Results of phenotypic identification had nearly identical sequence similarity based on comparison with the NCBI BLASTn database. Strain KKSSR91 (GenBank accession number: MN368306) exhibited a homology of 99\% with Rhodopseudomonas palustris YSC3 (CP019967.1).

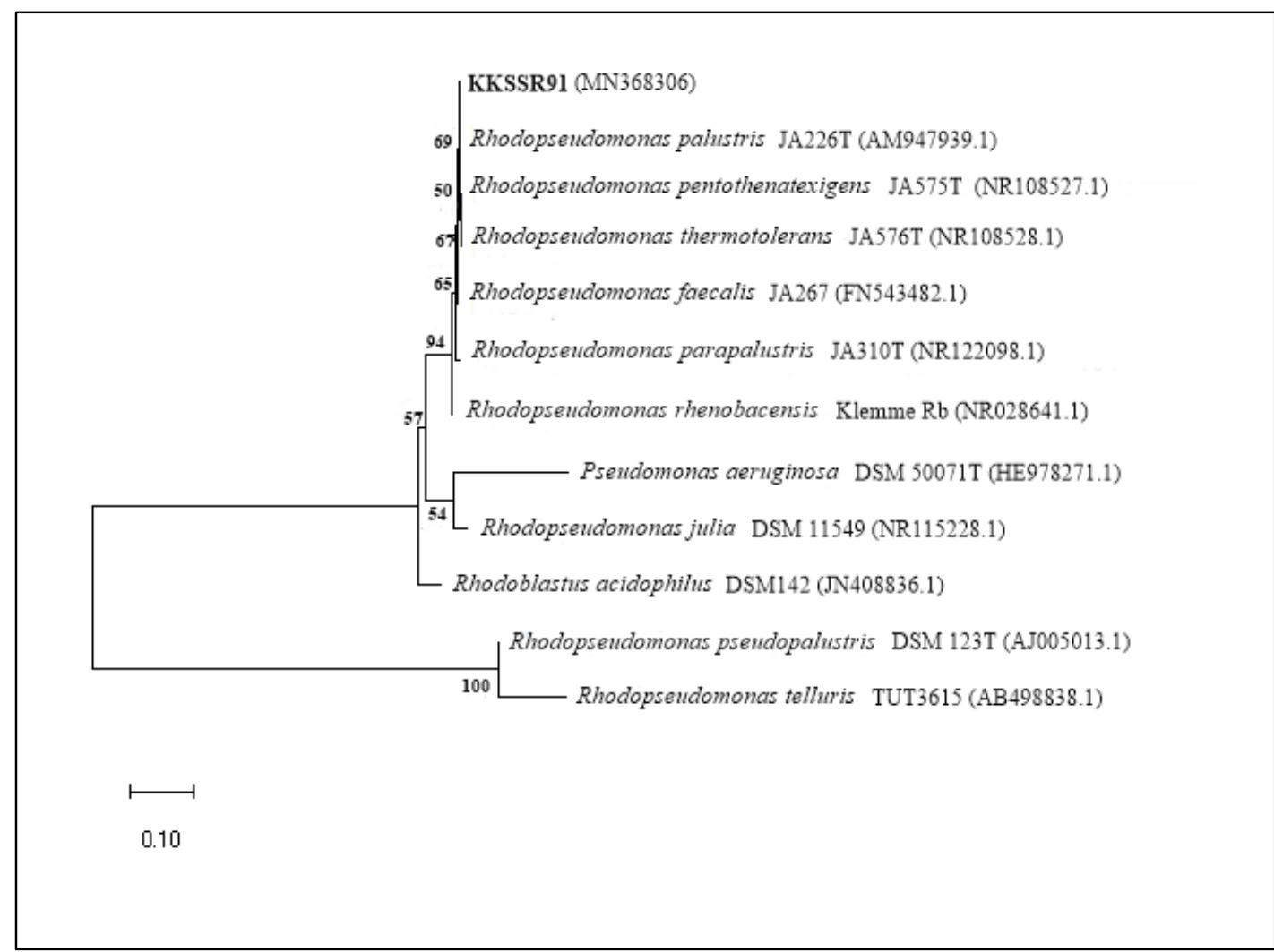

Figure 5. Evolutionary relationships of acid tolerant PNSB strain KKSSR91 (MN368306) withrepresentatives of the genus Rhodopseudomonas. The phylogenetic tree was constructed by the Maximum Likelihood (ML) method with MEGA-X and bootstrap consensus tree from 1,000 replicates; only value above $50 \%$ are given.

\section{DISCUSSION}

The acidic condition of soil is one of the most serious environmental stresses to affect the growth of crops and agricultural productivity. The treatment of seeds and seedlings with PGPB such as phototrophic bacteria has been proved to alleviate the effects of acidic soils (Khuong et al., 2018; Nookongbut et al., 2019). In anoxic acidic soils such as flooded paddy field, the problem of iron toxicity is caused by excessive $\mathrm{Fe}$ uptake due to $\mathrm{Fe}^{2+}$ from ferric $\left(\mathrm{Fe}^{3+}\right)$ reduction. Siderophores-producing bacteria could overcome this negative effect 
of acidic Fe rich soil (Stein et al., 2009). Moreover, siderophores produced by PNSB were able to mitigate the toxic effects of arsenic stress on seedling growth (Nookongbut et al., 2018). Since PNSB could produce siderophores in the present study, it should be possible to apply them to acid sulfate soils.

IAA and ALA were reported not only to promote plant growth but also to regulate plants spatially and help them adapt to abiotic stress (Blakeslee et al., 2019; Grobelak et al., 2019). Soil acidification affects the bioavailability of plant nutrients, especially inefficient nitrogen sources (Hao et al., 2019). This present study reported that $R$. palustris KKSSR91 was able to secrete IAA $(29.58 \mathrm{mg} / \mathrm{L})$ and ALA (2.89 mg/L) and fix $\mathrm{N}_{2}$ from its environment (Figure 4; Tables 1 and 2). ALA production by KKSSR91 grown in acid stress conditions in this study was lower than in other reports. However, low dosages of ALA (0.1-10 mg/L) were able to improve plant growth (Zhang et al., 2006). This suggests that this strain could be used as a PGPB in stress conditions for sustainable agriculture. In addition, ALA production by $R$. palustris KKSSR91 showed no sensitivity to light intensity in a range from 3,000 to 5,000 lux (Table 2); however, some strains of this genus such as TN114 and PP803 preferred a specific light intensity for ALA production (Nunkaew et al., 2018). This suggests that $R$. palustris KKSSR91 could be an effective PGPB in agricultural application.

In this study, the GI of kidney beans was used to evaluate the potential of R. palustris KKSSR91 to reduce the phytotoxic effect of acid $(\mathrm{pH}=4.5)$ on seed germination. Seeds germinated in biomass inoculated with this strain produced a GI index roughly $10 \%$ higher than the control (Table 3 ). This indicates that this strain could improve seed germination by increasing plant resistance to acidic stress. This finding is supported by the finding of Kantha et al., (2015), who reported that $R$. palustris $\mathrm{P} 1$ stimulated rice seed germination based on the GI index under salt stress. Koh and Song (2007) reported that IAA and ALA produced by Rhodopseudomonas sp. also enhanced germinated seeds.

Strain KKSSR91 was identified as $R$. palustris with $99 \%$ similarity to R. palustris YSC3 (Figure 5). R. palustris strains YSC3 and PS3 isolated from Taiwanese paddy soils produced different significant effects on plant growth and PS3was found to be more effective (Lo et al., 2018). Based on the results of seed germination, plant growth promotion by strain KKSSR91 was more similar to the behavior of strain PS3 than strain YSC3. It should be noted that only $1 \mathrm{~g}$ wet biomass/L was sufficient to promote seed germination under acidic stress (Table 3). Therefore, PNSB-inoculated biomass and seeds could potentially be mixed before planting in acid sulfate soils to increase seed proliferation for more sustainable agriculture. 
Table 3. Influence of inoculated PNSB strain KKSSR91 on germination index of kidney bean (Phasecolus vulgaris L.) under acid stress conditions.

\begin{tabular}{ccc}
\hline PNSB & \multicolumn{2}{c}{ Germination index (\%) } \\
KKSSR91 & pH 7.0 & pH 4.5 \\
Control & $100.00^{\mathrm{c}}$ & $58.68 \pm 25.37^{\mathrm{a}}$ \\
\cline { 2 - 3 } $1 \mathrm{~g} / \mathrm{L}$ & $84.87 \pm 20.57^{\mathrm{b}}$ & $68.81 \pm 20.47^{\mathrm{c}}$ \\
$5 \mathrm{~g} / \mathrm{L}$ & $51.41 \pm 18.12^{\mathrm{a}}$ & $61.92 \pm 17.10^{\mathrm{ab}}$ \\
$10 \mathrm{~g} / \mathrm{L}$ & $54.54 \pm 14.97^{\mathrm{a}}$ & $64.34 \pm 16.92^{\mathrm{b}}$ \\
\hline Note: & Value is mean of three replicates (one replicate $=10,3 \times 10=30) \pm$ standard deviation (S.D.); Control: no \\
& $\begin{array}{l}\text { PNSB inoculation as only distilled water. Different letters in each column indicate significant differences } \\
\text { at } P<0.05 .\end{array}$
\end{tabular}

\section{CONCLUSIONS}

Under acidic stress, four strains of PNSB showed potential as PGPB by producing the PGPS; ALA, IAA and siderophores and by releasing ammonium ion from $\mathrm{N}_{2}$ fixation. The most effective plant growth promoting strain, $R$. palustris KKSSR91, was found to significantly improve kidney bean seed germination under acidic stress. $R$. palustris KKSSR91 showed great potential to reduce acidic stress on plants based on its production of PGPS.

\section{ACKNOWLEDGEMENTS}

This research has been supported by the Center of Excellence on Biodiversity (BDC), Office of Higher Education Commission (BDC-PG1160002), and the first author has also been supported by the Postdoctoral Fellowship from the Prince of Songkla University, PSU. We thank the Research and Development Office (RDO), PSU.

\section{REFERENCES}

Alemu, H. 2017. Review paper on breeding common bean (Phaseolus Vulgaris L.) Genotypes for acidic soil tolerance. International Journal of Advanced Research and Publications. 1(3): 39-46.

Avissar, Y.J., and Moberg, P.A. 1995. The common origins of the pigments of life - early steps of chlorophyll biosynthesis. Photosynthesis Research, 44(3): 221-242. https://doi.org/10.1007/bf00048596

Blakeslee, J.J., Rossi T.S., and Kriechbaumer, V. 2019. Auxin biosynthesis: spatial regulation and adaptation to stress. Journal of Experimental Botany. 70(19): 5041-5049. https://doi.org/10.1093/jxb/erz283 
Bot, A.J., Nachtergaele, F.O., and Young, A. 2000. Land resource potential and constraints atregional and country levels. World Soil Resources Report 90. Rome: Food and Agricultural Organization of the United Nations. p.114.

Burnham, B.F. 1970. $\delta$-Aminolevulinic acid synthase (from Rhodopseudomonas sphaeroides). Methods in Enzymology.17A: 195-204

Gordon, S.A., and Weber, R.P. 1951. Colorimetric estimation of indole acetic acid. Plant Physiology. 26(1): 192-195. https://doi.org/10.1104/pp.26.1. 192

Grobelak, A., Świątek, J., Murtaś, A., and Jaskulak, M. 2019. Chapter 9 Cadmium-Induced Oxidative Stress in Plants, Cadmium Toxicity, and Tolerance in Plants: From Physiology to Remediation. In: Hasanuzzaman, M., Prasad M.N.V., and Fujita M. editors. Cadmium Toxicity and Tolerance in Plants from Physiology to Remediation. New York: Elsevier. p.213-231

Hao, T., Zhu, Q., Zeng, M. Shen, J., Shi, X., Liu, X., Zhang, F., and de Vries, W. 2019. Quantification of the contribution of nitrogen fertilization and crop harvesting to soil acidification in a wheat-maize double cropping system. Plant and Soil. 434: 167-184. https://doi.org/10.1007/s11104-018-3760-0

Kantachote, D., Nunkaew, T., Kantha, T., and Chaiprapat, S. 2016. Biofertilizers from Rhodopseudomonas palustris strains to enhance rice yields and reduce methane emissions. Applied Soil Ecology. 100: 154-161. https:// doi.org/10.1016/j.apsoil.2015.12.015

Kantha, T., Kantachote, D., and Klongdee, N. 2015. Potential of biofertilizers from selected Rhodopseudomonas palustris strains to assist rice (Oryza sativa L. subsp.indica) growth under salt stress and to reduce greenhouse gas emissions. Annals of Microbiology. 65: 2109-2118.

Khuong, N.Q., Kantachote, D., Onthong, J., and Sukhoom, A., 2017. The potential of acid-resistant purple nonsulfur bacteria isolated from acid sulfate soils for reducing toxicity of $\mathrm{Al}^{3+}$ and $\mathrm{Fe}^{2+}$ using biosorption for agricultural application. Biocatalysis and Agricultural Biotechnology. 12: 329-340. https://doi.org/10.1016/j.bcab.2017.10.022

Khuong, N.Q., Kantachote, D., Onthong, J. Xuan N.T., and Sukhoom, A. 2018. Enhancement of rice growth and yield in actual acid sulfate soils by potent acid-resistant Rhodopseudomonas palustris strains for producing safe rice. Plant and Soil. 429: 483-501. https://doi.org/10.1007/s11104-018-3705-7

Koh, R.H., and Song, H.G. 2007. Effects of Application of Rhodopseudomonas sp. on Seed Germination and Growth of Tomato Under Axenic Conditions. Journal of Microbiology and Biotechnology. 17(11): 1805-1810.

Lo, K.J., Lin, S.S., Lu, C.W., Kuo, C.H. and Liu, C.T. 2018. Whole-genome sequencing and comparative analysis of two plant-associated strains of Rhodopseudomonas palustris (PS3 and YSC3). Scientific Reports. 8: 12769. https://doi.org/10.1038/s41598-018-31128-8 
Madigan, M., and Jung, D., 2009. An overview of purple bacteria: systematics, physiology, and habitats, In: Hunter C.N., Daldal F., Thurnauer M., Beatty J.T. editors. The Purple Phototrophic Bacteria. Netherlands: Springer. p. 1-15.

Nguyen, K.Q., Kantachote, D., Onthong, J. and Sukhoom, A. 2018. Al ${ }^{3+}$ and $\mathrm{Fe}^{2+}$ toxicity reduction potential by acid-resistant strains of Rhodopseudomonas palustrisisolated from acid sulfate soils under acidic conditions. Annals of Microbiology. 68: 217-228.

Nookonbut, P., Kantachote, D., Khuong, N.Q., Sukhoom, A., Tantirungkij, M., and Limtong, S. 2019. Selection of acid-resistant purple nonsulfur bacteria from peat swamp forests to apply as biofertilizers and biocontrol Agents. Journal of Soil Science and Plant Nutrition. https://doi.org/10.1007/ s42729-019-00044-9.

Nookongbut, P., Kantachote, D., Megharaj, M., and Naidu, R. 2018. Reduction in arsenic toxicity and uptake in rice (Oryza sativa L.) by As-resistant purple nonsulfur bacteria. Environmental Science and Pollution Research. 25(36): 36530-36544. https://doi.org/10.1007/s11356-018-3568-8

Nunkaew, T., Kantachote, D., Kanzaki, H., Nitoda, T., and Ritchie, R.J. 2014. Effects of 5-aminolevulinic acid (ALA)-containing supernatants from selected Rhodopseudomonas palustris strains on rice growth under $\mathrm{NaCl}$ stress, with mediating effects on chlorophyll, photosynthetic electron transport and antioxidative enzymes. Electronic Journal of Biotechnology. 17: 19-26. https://doi.org/10.1016/j.ejbt.2013.12.004

Nunkaew, T., Kantachote, D., Nitoda, T. and Kanzaki. H. 2015a. Selection of salt tolerant purple nonsulfur bacteria producing 5-aminolevulinic acid (ALA) and reducing methane emissions from microbial rice straw degradation. Applied Soil Ecology. 86: 113-120. https://doi.org/10.1016/j.apsoil.2014. 10.005

Nunkaew, T., Kantachote, D., Nitoda, T., Kanzaki, H. and Ritchie, R.J. 2015 b. Characterization of exopolymeric substances from selected Rhodopseudomonas palustris strains and their ability to adsorb sodium ions. Carbohydrate Polymers. 115: 334-341. https://doi.org/10.1016/j. carbpol.2014.08.099

Nunkaew, T., Kantachote, D., Chaiprapat, S., Nitoda, T., and Kanzaki. H. 2018. Use of wood vinegar to enhance 5-aminolevulinic acid production by selected Rhodopseudomonas palustris in rubber sheet wastewater for agriculture use. Saudi Journal of Biological Sciences. 25(4): 642-650. https://doi.org/10.1016/j.sjbs.2016.01.028

Rangel, A.F., Mobin, M., Rao, I.M., and Horst, W.J. 2005. Proton toxicity interferes with the screening of common bean (Phaseolus vulgaris L.) genotypes for aluminum resistance in nutrient solution. Journal of Plant Nutrition and Soil Science. 168: 607-616. https://doi.org/10.1002/jpln. 200520509 
Sakpirom, J., Kantachote, D., Nunkaew, T., and Khan, E. 2017. Characterizations of purple non-sulfur bacteria isolated from paddy fields, and identification of strains with potential for plant growth-promotion, greenhouse gas mitigation and heavy metal bioremediation. Research in Microbiology. 168(3): 266-275. https://doi.org/10.1016/j.resmic.2016.12.001

Sasaki, K., Tanaka, T., Nishizawa, Y., Hayashi, M. 1990. Production of a herbicide, 5-aminolevulinic acid, by Rhodobacter sphaeroides using the effluent of swine waste from an anaerobic digestor. Applied Microbiology and Biotechnology, 32(6): 727-731. https://doi.org/10.1007/BF00164749.

Stein, R.J., Duarte, G.L., Spohr, M.G., Lopes, S.I.G., and Fett, J.P. 2009. Distinct physiological responses of two rice cultivars subjected to iron toxicity under field conditions. Annals of Applied Biology.154(2): 269-277. https://doi.org/10.1111/j.1744-7348.2008.00293.x

Yang, Z.B., Rao, I.M., and Horst, W.J. 2013. Interaction of aluminum and drought stress on rootgrowth and crop yield on acid soils. Plant and Soil. 372: 3-25. https://doi.org/10.1007/s11104-012-1580-1

Zhang, Z.J., Li, H.Z., Zhou, W.J., Takeuchi, Y., and Yoneyama, K. 2006. Effect of 5-aminolevulinic acid on development and salt tolerance of potato (Solanum tuberosum L.) microtubers in vitro. Plant Growth Regulation. 49: 27-34. https://doi.org/10.1007/s10725-006-0011-9 